\title{
Shield design for the XENON1T experiment at LSM
}

\author{
Cecilia LEVY*† \\ Subatech \\ E-mail: cecilia.levy@subatech.in2p3.fr
}

The direct search for dark matter experiment, XENON100, using liquid xenon, has been in the process of taking data since January 2010 and will continue to do so for another year to reach a never yet achieved sensitivity on the WIMP nucleon cross section and may ultimately detect WIMPs. In addition, the experiment is already being pushed towards its next phase, XENON1T, which will be much larger with a fiducial mass of about 1 ton of liquid xenon. Reducing the background, it should be a hundred times more sensitive. While the previous phases were located at the Laboratori Nazionali del Gran Sasso (LNGS), in Italy, XENON1T could be hosted in the Laboratoire Souterrain de Modane (LSM), in France. The LSM site and its different level of gamma, neutron and muon background with an emphasis on the muon induced neutrons coming from the environmental rocks, have been investigated. Considering the available space at LSM and the final background level necessary for a dark matter search, an efficient and optimal shielding design has been established. All the results of these studies will be presented hereafter.

Identification of Dark Matter 2010

July 26 - 302010

University of Montpellier 2, Montpellier, France

*Speaker.

† On behalf of the XENON Collaboration 


\section{Introduction}

The universe, as we know it, is made of only $5 \%$ of visible matter. Out of the remaining $95 \%, 25 \%$ would be constituted of what is called dark matter. One of the most seducing theories that includes dark matter, supersymmetry, predicts that it is made of Weakly Interacting Massive Particles (WIMP). Direct search experiments have been set up to try to directly detect the WIMPs by looking at the scintillation, ionization or heat signal from the recoil of a target nucleus. The XENON experiment is one such, which uses liquid xenon as a scintillation and ionization target.

\section{The XENON1T Experiment}

XENON1T is the next step of the XENON experiment. The detector as shown on figure 1(a), is a two-phase time projection chamber (TPC) filled with xenon that exploits many of the design features validated with XENON100 [1].

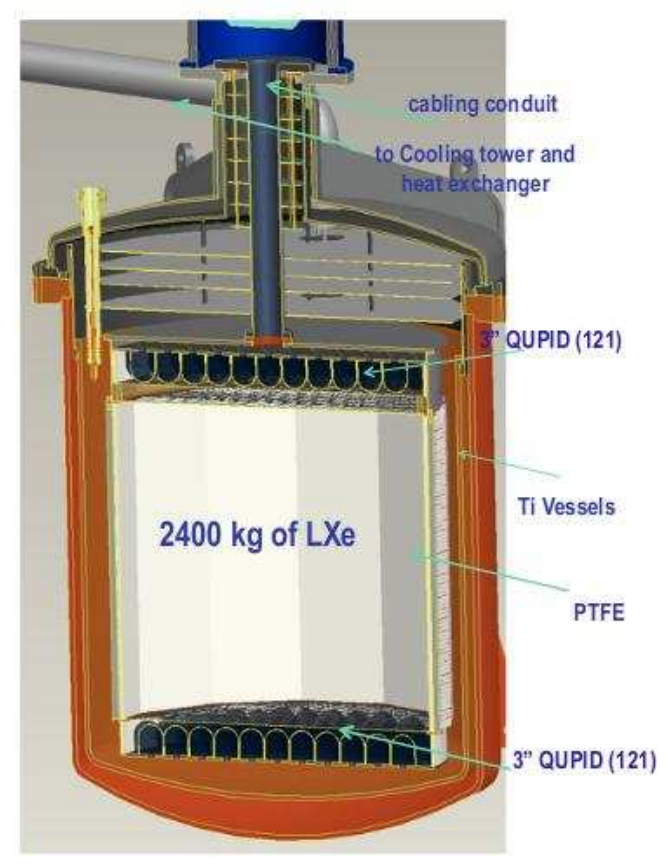

(a) The XENON1T detector

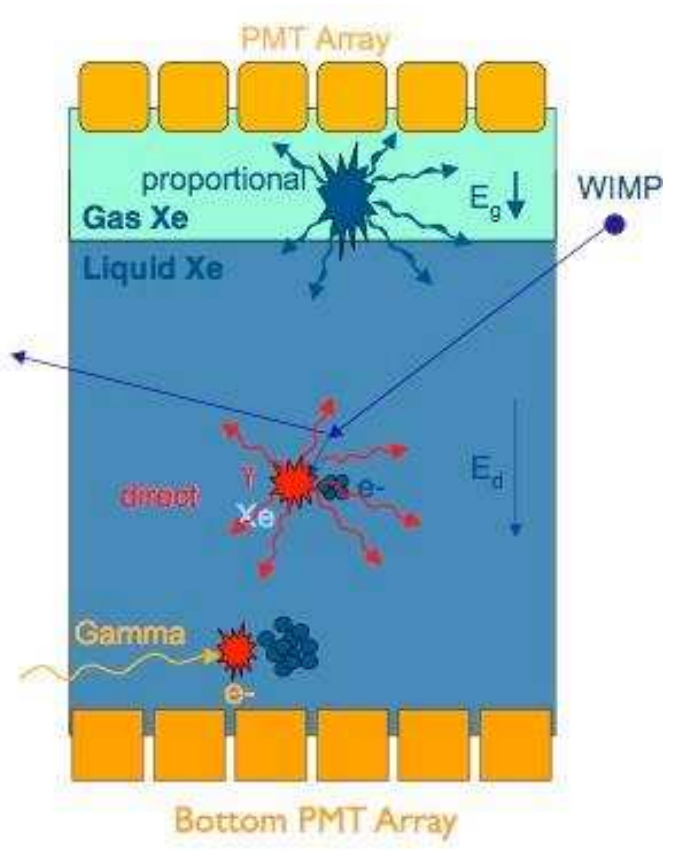

(b) Principle of a XENON detector

Figure 1: The XENON detector

Like its predecessor, XENON1T will have the same detection principle by looking at both ionization and scintillation signals as shown on figure 1(b). When a WIMP or another particle hits a liquid xenon atom, the energy is split into nuclear excitation and ionization. When the atom goes back to its stable state it emits scintillation light. These photons are detected by photodetectors and gives a first signal $S_{1}$. Meanwhile the ionized electrons are drifted in the TPC until they reach a layer of gaseous xenon where they produce a secondary scintillation which is detected by the arrays of photodetectors, producing a second signal $S_{2}$.

The ratio of $S_{2} / S_{1}$ allows the discrimination between electronic and nuclear recoils, and thus between gammas and neutrons or WIMPs. Alpha particles are of no concern as they are not pen- 
etrative enough and the volume of xenon acts as a shield. To reduce the neutron background, the experiment is deep underground and diverse shields are used such as lead, polyethylene, water or copper.

XENON1T will have several improvements over XENON100. Its internal background will be reduced by the use of lower radioactivity vessels and photodetectors (QUPIDs), as well as by the use of ultra pure liquid with a ${ }^{85} \mathrm{Kr} / \mathrm{Xe}$ contamination at sub-ppt level. Event localization in 3D, self-shielding by LXe and adequate shielding from external gamma and neutron backgrounds will enable an ultra low background rate in a fiducial volume of about one ton. The projected sensitivity reach for the spin-independent WIMP-nucleon cross section for $100 \mathrm{GeV}$ WIMPs is better than $10^{-46} \mathrm{~cm}^{2}$.

\section{Location and Shield Design}

While XENON100 is located at Laboratori Nazionali del Gran Sasso (LNGS) in Italy, XENON1T's location is still undetermined. While it could stay at LNGS, another possible option is to set it as shown on figure 2(a) at the Laboratoire Souterrain de Modane (LSM) in France where, due to its increased depth compared to LNGS, it would benefit from a 4 times lower cosmic ray flux and a radon free facility as can be seen on tables 1 and 2. Due to the constraints of space, if XENON1T were to be built at LSM, the only available shielding option is a solid shield with successive layers of polyethylene and lead as shown on figure 2(b), so that it is efficient against the most relevant backgrounds.

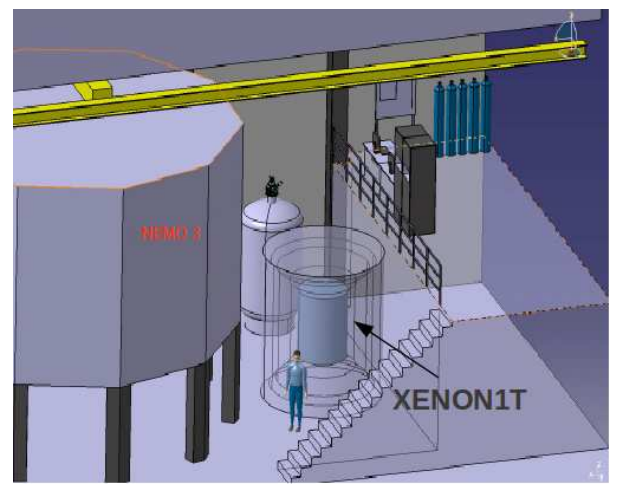

(a) Location of the XENON1T experiment at LSM

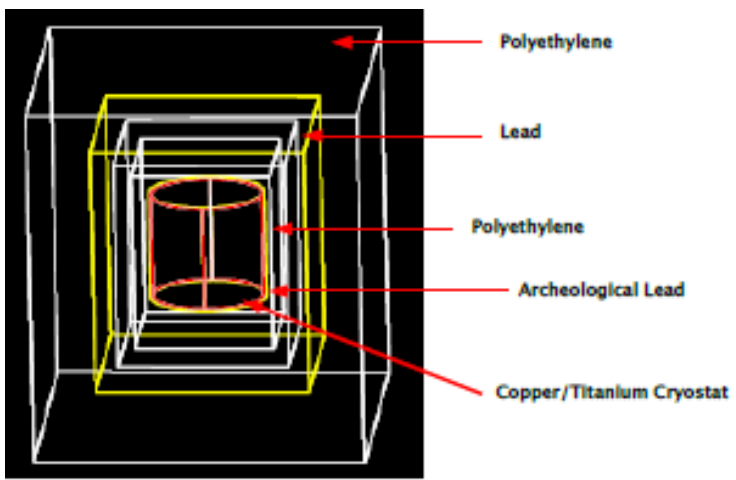

(b) Shield design of the XENON1T experiment at LSM

Figure 2: Location and shielding structure of the XENON1T experiment at LSM

\begin{tabular}{|c|c|c|c|c|c|}
\hline Lab & $\begin{array}{c}\text { Depth } \\
(\mathrm{m} . w . e)\end{array}$ & $\begin{array}{c}\text { Equivalent vertical [3] } \\
\text { depth (m.w.e.) }\end{array}$ & $\begin{array}{c}\text { Muon flux } \\
\left(\mathrm{m}^{-2} \mathrm{~d}^{-1}\right)\end{array}$ & $\begin{array}{c}\text { Mean energy } \\
\text { neutron flux }(\mathrm{GeV})\end{array}$ & $\begin{array}{c}\text { Muon induced } \\
\left(\mathrm{m}^{-2} \mathrm{~d}^{-1}\right)\end{array}$ \\
\hline LSM & $\approx 4800$ & $\approx 4200$ & $4.17 \pm 0.43$ & $\approx 300$ & 0.536 \\
LNGS & $\approx 3750$ & $\approx 3100$ & $22.3 \pm 2.6$ & $\approx 270$ & 2.35 \\
\hline
\end{tabular}

Table 1: Muon flux and total muon induced neutron flux at the rock/cavern boundary in LSM [4] [3] and LNGS [3] 


\begin{tabular}{|c|c|c|}
\hline LSM & $\begin{array}{c}\text { Natural } \\
15 \mathrm{~Bq} / \mathrm{m}^{3}\end{array}$ & $\begin{array}{c}\text { Radon-free air } \\
18 \mathrm{mBq} / \mathrm{m}^{3}\end{array}$ \\
\hline LNGS & $\mathrm{XeBox}$ & Inside Shield \\
& $200-700 \mathrm{~Bq} / \mathrm{m}^{3}$ & $1-2 \mathrm{~Bq} / \mathrm{m}^{3}$ \\
\hline
\end{tabular}

Table 2: Radon rates

An external $55 \mathrm{~cm}$ thick layer of polyethylene is first implemented to shield against muon induced neutrons as well as neutrons from ${ }^{238} \mathrm{U},{ }^{235} \mathrm{U}$ and ${ }^{232} \mathrm{Th}$ decays in the rock and the concrete. An inner $20 \mathrm{~cm}$ thick lead layer is then added to shield for the gammas from ${ }^{238} \mathrm{U},{ }^{232} \mathrm{Th}$ and ${ }^{40} \mathrm{~K}$ decays in the rock and the concrete, and from those from ${ }^{238} \mathrm{U},{ }^{232} \mathrm{Th},{ }^{60} \mathrm{Co}$ and ${ }^{40} \mathrm{~K}$ in the outer polyethylene layer. This is followed by a second $15 \mathrm{~cm}$ thick inner polyethylene layer to shield against the neutrons from ${ }^{210} \mathrm{~Pb},{ }^{238} \mathrm{U}$ and ${ }^{232} \mathrm{Th}$ decays in the lead layer. Finally an inner $2 \mathrm{~cm}$ thick cylindrical layer of archaeological lead with very low intrinsic activity is put into place to shield for the gammas from the inner polyethylene layer. In addition to this passive shield, an active muon veto would allow to reject all events in coincidence with a muon.

\section{Monte-Carlo Simulations}

The MC simulations are done using GEANT4.9.1.p03. The software SOURCES was used to generate the neutron spectra from $\alpha$-n reactions and spontaneous fission due to ${ }^{238} \mathrm{U}$ and ${ }^{232} \mathrm{Th}$ in different materials. The generation of the neutrons is done by propagating the muons through the rock, following all the neutrons produced by direct spallation, electromagnetic or hadronic cascades and recording their entrance point in the cavern, momentum and energy. This was done for $5 \mathrm{~m}$ of LNGS rock but is applicable to LSM which has roughly the same rock and concrete composition. Neutrons are the focus of this study as they interact via nuclear recoils mimicking a dark matter signal. Muon-induced neutrons generated inside the detector are not considered in this study since the muon veto will allow to reject them.

As shown on figure 3(a), the dominant background comes from the muon induced neutrons from the rock and the concrete. As shown on figure 3(b), the muon induced neutron spectrum is mainly at high energies which reduces considerably the influence of the polyethylene on these neutrons. The intrinsic neutron background shown on figure 3(a) is linear due to the shielding power of the polyethylene on itself. As for the neutron flux due to the lead after $15 \mathrm{~cm}$ of polyethylene, it increases with the lead thickness as expected, but is still negligible compared to the muon induced neutrons.

Table 3 shows the resulting flux of muon induced neutrons inside the detector with a fiducial volume of $1 \mathrm{~T}$ and recoil energies between 0 and $100 \mathrm{keV}$. Adding a total of $70 \mathrm{~cm}$ of polyethylene (55 cm external and $15 \mathrm{~cm}$ internal) reduces the rate by a factor 3 compared to only $35 \mathrm{~cm}$ of polyethylene, yielding a muon induced rate below $0.1 \mathrm{n} / \mathrm{yr} / \mathrm{T}$. Considering the space available at LSM, $70 \mathrm{~cm}$ is judged to be an acceptable thickness of polythylene from all stand points.

The dominant contribution to the $\gamma$ background comes from the concrete, the flux from the polyethylene being 200 times smaller thus negligible in comparison. Table 4 gives the rate of single scatters due to gammas in the 1T fiducial volume. 


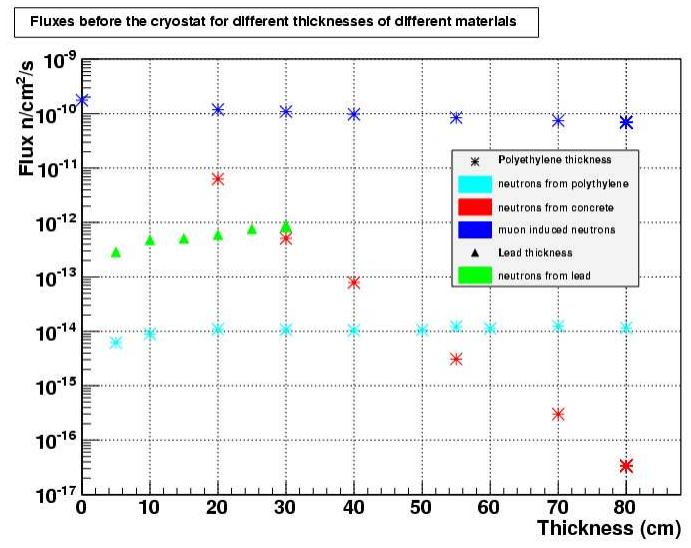

(a) Neutron background

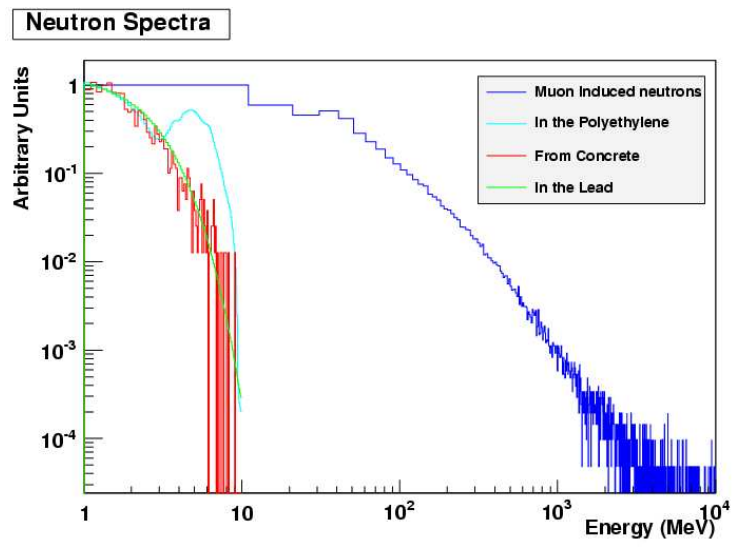

(b) Neutron spectra

Figure 3: Neutron background and spectra from different shielding elements

\begin{tabular}{|c|c|}
\hline $\begin{array}{c}\text { Total Polyethylene Thickness } \\
\text { External + Internal }(\mathrm{cm})\end{array}$ & $\begin{array}{c}\text { Neutron flux } \\
(\mathrm{nr} / \mathrm{yr} / \mathrm{T})\end{array}$ \\
\hline $20+15$ & $0.1 \pm 0.02$ \\
$40+15$ & $0.09 \pm 0.02$ \\
$55+15$ & $0.04 \pm 0.01$ \\
$70+15$ & $0.04 \pm 0.02$ \\
\hline
\end{tabular}

Table 3: Neutron background from single step nuclear recoil between 0 and $100 \mathrm{keV}$ in the 1 tonne fiducial volume

\begin{tabular}{|c|c|}
\hline $\begin{array}{c}\text { Pb Thickness } \\
(\mathrm{cm})\end{array}$ & $\begin{array}{c}\text { Gamma flux } \\
\text { (single scatter/yr/T) }\end{array}$ \\
\hline 5 & $0.001 \pm 0.004$ \\
10 & $<0.0001$ \\
20 & $<6 \mathrm{e}-5$ \\
30 & $<2 \mathrm{e}-5$ \\
\hline
\end{tabular}

Table 4: Gamma background in the 1 tonne fiducial volume

Since, like in XENON100, the S2/S1 discrimination allows greater than 99\% rejection of electron recoils, it is clear that $20 \mathrm{~cm}$ of lead is enough for the gamma background to be lower than $1 \mathrm{event} / \mathrm{yr} / \mathrm{T}$ in the fiducial volume in the dark matter region for recoil energies between 0 and 100 $\mathrm{keV}$.

\section{Conclusion}

By locating XENON1T at LSM and using a multi layer lead and polyethylene shield, a background of less than 0.1 nuclear recoil /yr/T is reachable. This will allow to perform a one year background free experiment to reach a sensitivity better than $10^{-46} \mathrm{~cm}^{2}$. Thanks to a deeper site, the proposed shield design is equivalent to a 3 meter water shield at LNGS. 


\section{References}

[1] Xenon100 Collaboration, E. Aprile et al., First dark matter results from the xenon 100 experiment Physical Review Letters, Vol. 105, No. 13. (September 2010)

[2] A. Nachab and the NEMO Coll., Radon reduction and Radon monitoring in the NEMO experiment AIP Conf. Proc. 897, 35 (2007)

[3] D.-M. Mei and A. Hime, Muon-induced background study for underground laboratories [Physical Review D 73 (2006)]

[4] L. Chabert, PhD Thesis: Etude du bruit de fond neutron induit par les muons dans l'experience EDELWEISS II, July 2004 (in French) (http://edelweiss.in2p3.fr/pub/fichiers/theses.html) 